\title{
Solvent-free Catalysed Synthesis of Tetrahydropyran Odorants: the Role of $\mathrm{SiO}_{2} \cdot p$-TSA Catalyst on the Prins-Cyclization Reaction
}

\author{
Alexandra Macedo, ${ }^{a}$ Edison P. Wendler, ${ }^{a}$ Alcindo A. Dos Santos, ${ }^{*, b}$ \\ Julio Zukerman-Schpector*,a and Edward R. T. Tiekink ${ }^{c}$ \\ ${ }^{a}$ Departamento de Química, Universidade Federal de São Carlos, Rod. Washington Luís km 235, \\ 13565-905 São Carlos-SP, Brazil \\ ${ }^{b}$ Instituto de Química, Universidade de São Paulo, Av. Prof. Lineu Prestes 748, \\ 05508-000 São Paulo-SP, Brazil \\ 'Department of Chemistry, University of Malaya, 50603 \\ Kuala Lumpur, Malaysia
}

\begin{abstract}
Foi desenvolvido um procedimento eficiente e ecologicamente amigável para a promoção da ciclização de Prins em ausência de solventes orgânicos baseado na maceração de um aldeído e um álcool alílico em presença de quantidade catalítica de $p$-TSA disperso em sílica gel. Por esse procedimento foram sintetizadas as fragrâncias comerciais Florol ${ }^{\circledR}$ e Clarycet $^{\circledR}$, em uma e duas etapas sintéticas respectivamente.
\end{abstract}

An efficient, green and solvent-free catalysed Prins-cyclization reaction based on the simple grinding of an aldehyde and a homoallylic alcohol in the presence of catalytic amount of $p$-TSA on silica gel is reported. By this protocol were synthesized tetrahydropyran odorants including commercial Florol $^{\circledR}$ and Clarycet ${ }^{\circledR}$, in one and two steps respectively.

Keywords: Prins-cyclisation, solvent-free catalysis, green chemistry, tetrahydropyrans, fragrances

\section{Introduction}

The Prins-cyclization reaction is one of the most efficient strategies to construct the tetrahydropyran core, ${ }^{1}$ which is present in several natural products. ${ }^{2}$ Some non-natural compounds possessing this functionality were found to be of commercial interest by the perfume industry. Clarycet $^{\oplus}$ (commercialized by IFF) and Florol $^{\circledR}$ (commercialized by Firmenich) are floral odorants handled in the industry as a racemic diastereoisomeric mixture which can be used in a large number of fragrances and formulations conferring floral scents without changing the olfactory character of the perfume. ${ }^{3,4}$ So far as we know, there are only two works in the literature describing the synthesis of both, Clarycet ${ }^{\circledR 4}$ and Florol ${ }^{\oplus}$. In one of these works, racemic Florol ${ }^{\circledR}$ was synthesized in $44 \%$ overall yield from geraniol/nerol mixture in 5 sequential steps. All enantioenriched stereoisomers of

*e-mail: alcindo@iq.usp.br both odorants were prepared by an enzymatic approach in 6 sequential steps in very low yields. ${ }^{4}$

With the recent increase of environmental awareness and subsequent development of "green-chemistry" principles, several guidelines for the production of chemicals by cleaner and benign procedures are being established as crucial for the modern chemical industry processes. One of the most important requirements in this context is the development of alternative synthetic routes aimed at minimizing or substituting mineral solvents by more benign solvents such as water, acetone, ethanol, ethyl acetate, etc. ${ }^{5}$ Further, emerging solvent-free processes and the utilization of solid matrices that can act as dispersing agents or as catalysts that is attracting attention. ${ }^{6}$

In this work, a one-pot procedure for the construction of the tetrahydropyran core of the floral odorants, Clarycet ${ }^{\circledR}$, Florol $^{\circledR}$ and other two not yet commercially utilized octahydro- $2 H$-chromen-4-ols ${ }^{7}$ using a solvent-free solid phase catalysed Prins-cyclization reaction is presented. 


\section{Results and Discussion}

This proposition was based on previous work related to the solvent-free solid phase catalysed tetrahydropyranylation of alcohols and thiols. ${ }^{8}$ Earlier, it was observed that citronellal can be converted, in $91 \%$ isolated yield, into iso-pulegol within 2 min by an intramolecular ene-type reaction. The reaction proceeds by grinding it in a mixture of silica gel with catalytic amount of $p$-toluenosulfonic acid ( $p$-TSA) according to Scheme 1.

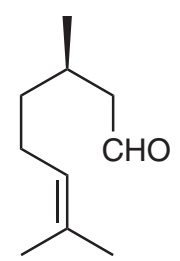

1: (+)-citronellal

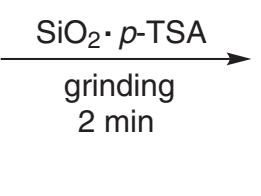

2: iso-pulegol (91\%)
Scheme 1. Synthesis of iso-pulegol by catalytic system.

This result stimulated the application of similar reaction conditions in the preparation of tetrahydropyran derivatives by the intermolecular version of the Prins-cyclization reaction and studies related of conformational features of three tetrahydropyrans. ${ }^{9}$ A detailed stoichiometric screen showed that a physical mixture of $p$-TSA $(0.29 \mathrm{mmol})$ in silica gel $(0.5 \mathrm{~g})$ was the best dispersant/catalyst system in the conversion of 3-methyl-but-3-en-1-ol (3,1 mmol) and benzaldehyde $(4,1.1 \mathrm{mmol})$ in the corresponding Prins adduct 5, (syn:anti, 63:37) in 88\% isolated yield, after 5 min grinding as presented in Scheme 2.<smiles>C=C(C)CCO</smiles>

3
5: $88 \%(63: 37)$
syn : anti

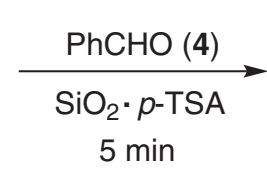

Scheme 2. Synthesis of model compound $\mathbf{5}$.

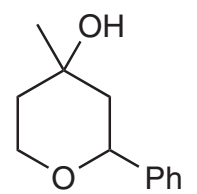

In order to investigate the role of the $\mathrm{SiO}_{2} / p$-TSA mixture, both components of this catalytic system, $\mathrm{SiO}_{2}$ and $p$-TSA, were investigated separately in the reaction of 3 with 4 . $\mathrm{SiO}_{2}$ only did not display any catalytic behaviour even at long reaction times. On the other hand when sub-stoichiometric amount of $p$-TSA was put together with $\mathbf{3}$ and $\mathbf{4}$ in absence of solvents, a very vigorous and exothermic reaction took place accompanied by partial carbonization of the components of the mixture, evidenced by the formation of a black colour and the title compound was produced in low yield among many other by-products. Other solid matrices (neutral, acid and basic aluminium oxide, Celite ${ }^{\circledast}$ as well Montmorillonite K10) were also investigated as partners of $p$-TSA in the reaction of $\mathbf{3}$ with $\mathbf{4}$ and failed to produce $\mathbf{5}$ in good yields and synthetic useful reaction time in comparison with $\mathrm{SiO}_{2}$. These experiments allow us to conclude that $\mathrm{SiO}_{2}$ in association with $p$-TSA acts as catalyst and not only as a dispersant agent. In this way, were adopted the reaction conditions presented in Scheme 2, as the ideal system for the preparation of the Prins adducts by a solvent-free protocol in the reaction of the alcohol $\mathbf{3}$ with other aldehydes as presented in Scheme 3 and summarized in Table 1.

In all cases the tetrahydropyran derivatives were formed, as a diastereoisomeric mixture, in reasonable to good yields by the simple grinding of the reagents in a mixture of silica gel and catalytic amount of $p$-TSA. While aldehydes $\mathbf{6 a}, \mathbf{6 c}$ and $\mathbf{6 g}$, as well as benzaldehyde $\mathbf{4}$, were converted to the corresponding products in good yields in only 5 min of reaction (entries 1, 3, 7 and Scheme 2), the aromatic $\mathbf{6 b}, \mathbf{6 d}$, cycloalkyl $\mathbf{6 f}$ and alkenyl $\mathbf{6 h}$ aldehydes required a longer reaction time for conversion to the corresponding products (entries 2, 4, 6 and 8). Aldehyde 6e required $25 \mathrm{~min}$ to be converted into the product $7 \mathbf{e}$ in $69 \%$ yield (entry 5 ).

The structure of the tetrahydropyran derivative $\mathbf{7 f}$ (major diastereoisomer) was subsequently confirmed to have the syn relation, by a single-crystal X-ray structure determination, as shown in Figure 1. ${ }^{10}$

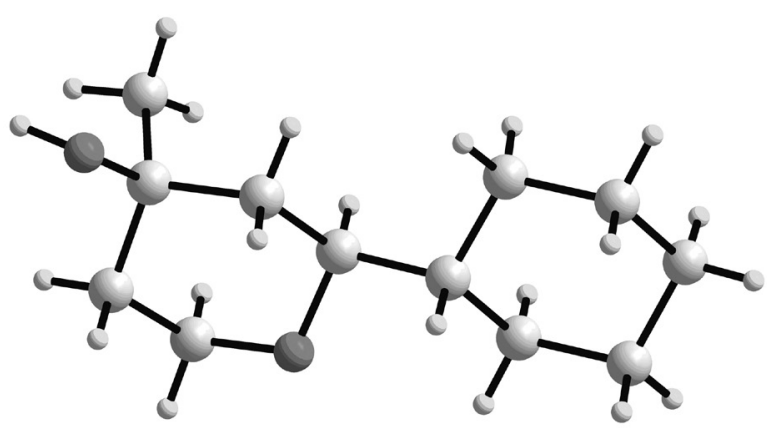

Figure 1. Molecular structure of $\mathbf{7 f}$ determined by X-ray crystallography.

The major diastereoisomer of $\mathbf{7 h}$ was submitted to nOe experiment and the syn relation was confirmed, as show in Figure 2.

The strong 1D nOe for methyl group and carbinolic hydrogen provided unquestionable proof for the assigned syn relation. On the basis of nOe experiment acquired for compound $\mathbf{7 h}$, the relation of the others tetrahydropyrans $\mathbf{5 , 7 a - g}$ was similarly assigned. 
With the reaction conditions established, this method was applied to the synthesis of Florol ${ }^{\circledR}(\mathbf{1 1}$, in a one-pot

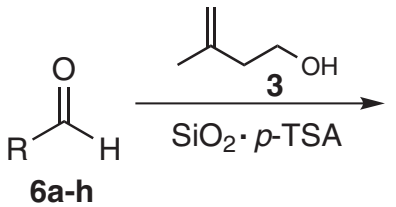<smiles>BC1CC(C)(O)CCO1</smiles>

7a-h

Scheme 3. Reaction of different aldehydes with compound 3 . procedure $87 \%, 15 \mathrm{~min})$ and Clarycet $^{\circledR}(\mathbf{1 2})$ by reacting alcohol $\mathbf{3}$ with the appropriate aldehydes (8 and $\mathbf{9 ,}$ respectively), and two other odorants of commercial interest $^{5}$ (15 and 16) by reacting iso-pulegol 2 with aldehydes 13 and 14, respectively (Scheme 4).

Despite the low yield of $\mathbf{1 5}$ (49\%), due the high volatility of aldehyde 13 , since the reactions were conduced by grinding the reagents in an open mortar, the synthesis can be considered attractive since it can be performed in a single step and by a solvent-free procedure.

Table 1. Prins-cyclization reaction of $\mathbf{3}$ with aldehydes $\mathbf{6 a - h}$

\begin{tabular}{|c|c|c|c|c|c|}
\hline Entry & Aldehyde Structure & Compounds & Products $^{\mathrm{a}}$ & time $(\min )^{\mathrm{b}}$ & Yield $\%{ }^{\mathrm{c}}\left(\right.$ syn:anti) ${ }^{\mathrm{d}}$ \\
\hline 1 & & $6 a$ & $7 a$ & 5 & $91(64: 36)$ \\
\hline 2 & & $6 \mathbf{b}$ & $7 b$ & 10 & $67(20: 80)$ \\
\hline 3 & & $6 c$ & $7 c$ & 5 & $89(30: 70)$ \\
\hline 4 & & 6d & $7 d$ & 40 & $70(55: 45)$ \\
\hline 5 & & $6 e$ & $7 e$ & 25 & $69(60: 40)$ \\
\hline 6 & & $6 f$ & $7 f$ & 60 & $71(62: 38)$ \\
\hline 7 & & $6 \mathrm{~g}$ & $7 g$ & 5 & $62(40: 60)$ \\
\hline 8 & & $6 h$ & $7 \mathrm{~h}$ & 10 & $58(71: 29)$ \\
\hline
\end{tabular}

${ }^{\mathrm{a} C}$ Compounds $\mathbf{7 a - h}$ as well a $\mathbf{5}$, were formed as a diastereisomeric mixture; ${ }^{\mathrm{b}}$ total grinding time; cisolated yields; ${ }^{\mathrm{d}} \mathrm{GC}$-FID and nOe experiments were used to determine the syn:anti ratio.
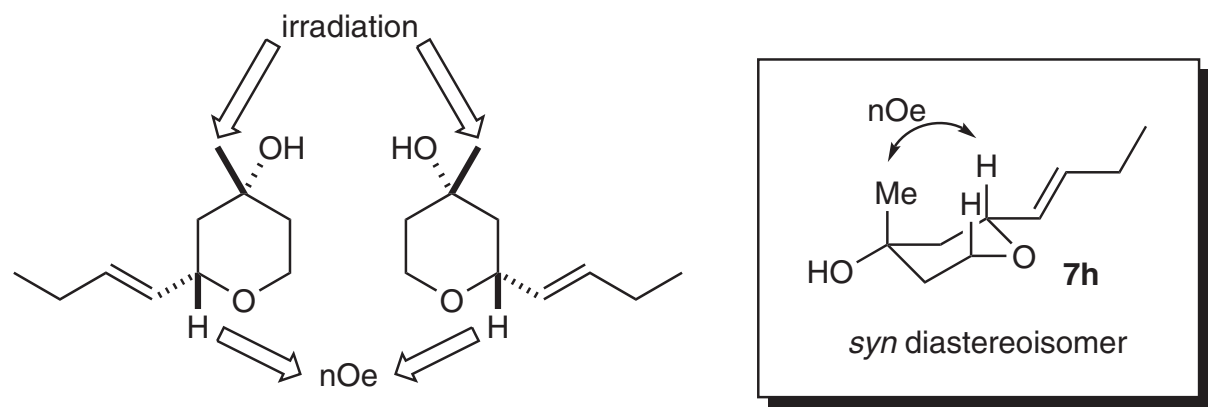

$$
(2 S, 4 R)+(2 R, 4 S)-7 \mathbf{h}
$$

Figure 2. Relative stereochemistry of $\mathbf{7 h}$ determined by nOe experiments. 

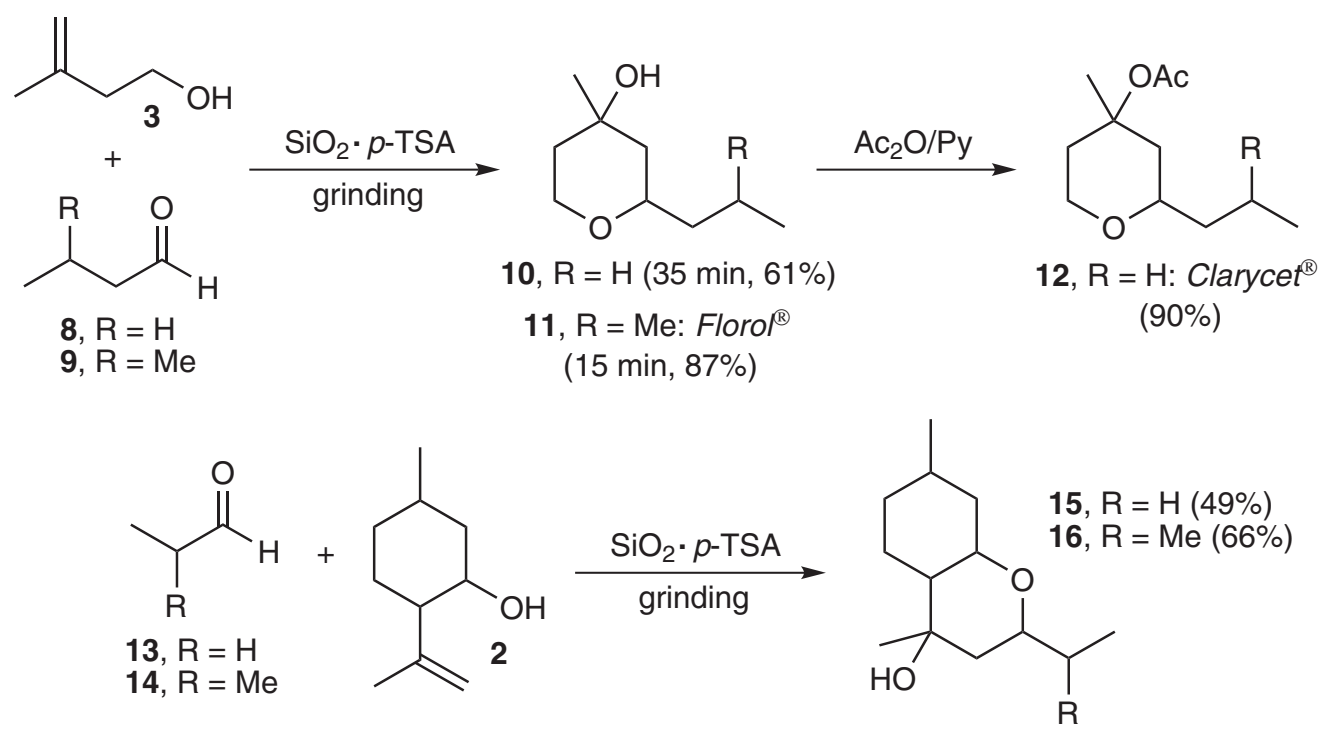

Scheme 4. Application of $\mathrm{SiO}_{2} \cdot p$-TSA system on synthesis of odorants with commercial interest.

\section{Conclusion}

In conclusion, a one-pot strategy has been developed and applied to the synthesis of three important odorants: Florol ${ }^{\circledR}$ and compounds $\mathbf{1 5}$ and $\mathbf{1 6}$. The synthesis of Clarycet $^{\oplus}$ was accomplished by a usual acetylation of the Prins adduct 10, with $55 \%$ overall yield. The great appeal of this procedure is found in its operational practicality, since the simple mixture of the reagents in a mortar allows the preparation of the target compounds in a few minutes. Currently, some variants of this protocol are under investigation aimed towards the total synthesis of industrially important compounds.

\section{Experimental}

\section{General}

The following includes general experimental procedures, specific details for representative reaction, and isolation and spectroscopic information for the new compounds prepared. ${ }^{1} \mathrm{H}$ and ${ }^{13} \mathrm{C}$ NMR spectra were recorded on a Bruker DRX-400 $400 \mathrm{MHz}$. Infrared spectra were recorded on a FT-IR Bomem-Hartman \& Braun MB-102. Low resolution mass spectra were obtained on a GC-17A Shimadzu, equipped with HP-5MS column (5\% phenyl / $95 \%$ dimethyl polysiloxane, $30 \mathrm{~m} \times 0.25 \mathrm{~mm} \times 0.25 \mu \mathrm{m})$, coupled with a GCMS-QP5000 Shimadzu $(70 \mathrm{eV})$.

High resolution mass spectra were obtained on a LC-MS Bruker Daltonics MicroTOF Ic by direct infusion. Analytical thin-layer chromatography (TLC) was performed on glass plates coated with $0.50 \mathrm{~mm} 230-400$ mesh silica gel containing a fluorescent indicator (Merck). TLC plates were visualized by exposure to ultraviolet light ( $254 \mathrm{~nm})$ and/or by immersion in an acidic staining solution of $p$-anisaldehyde followed by heating on a hot plate. Organic solutions were concentrated by rotary evaporation at $c a$. 30$100 \mathrm{mmHg}$. Flash column chromatography was performed on Fluka silica gel 60 (220-440 mesh).$^{10}$ Solvents used were of commercial grade and were previously treated according to the literature. ${ }^{11}$

\section{General Procedure}

In a mortar containg silica gel (200-400 mesh) $(0.5 \mathrm{~g}$, $8.33 \mathrm{mmol})$ and $p$-toluenesulfonic acid $(0.05 \mathrm{~g}, 0.29 \mathrm{mmol})$ was added the aldehyde $(1 \mathrm{mmol})$ and homoallylic alcohol $(1.1 \mathrm{mmol})$. The resulting mixture was ground for the appropriate time and the reaction was monitored by thin layer chromatography. The reaction media was directly purified by column chromatography by elution with appropriate mixture of solvents.

\section{(5R)-5-Methyl-2-(prop-1-en-2-yl)cyclohexanol (2)}

The product was analysed by CG-FID and compared with commercial standard of iso-pulegol purchased from Aldrich Chemical Company (Milwaukee, Wisconsin, USA) (CAS 628693-74-3).

\section{4-Methyl-2-phenyltetrahydro-2H-pyran-4-ol (5)}

Syn diastereoisomer: Eluent: hexane/ethyl acetate (1:1), Rf 0.66; colorless oil, yield 55\%; ${ }^{1} \mathrm{H}$ NMR $(400 \mathrm{MHz}$, $\left.\mathrm{CDCl}_{3}\right) \delta 1.45(\mathrm{~s}, 3 \mathrm{H}), 1.66(\mathrm{~s}, \mathrm{OH}), 1.68\left(\mathrm{dq}, J_{1} 12.94 \mathrm{~Hz}\right.$, $\left.J_{2} 2.39 \mathrm{~Hz}, 1 \mathrm{H}\right), 1.72-1.86(\mathrm{~m}, 2 \mathrm{H}), 1.89\left(\mathrm{dt}, J_{1} 12.94 \mathrm{~Hz}\right.$, $\left.J_{2} 2.39 \mathrm{~Hz}, 1 \mathrm{H}\right), 3.62\left(\mathrm{td}, J_{1} 12.48 \mathrm{~Hz}, J_{2} 2.31 \mathrm{~Hz}, 1 \mathrm{H}\right), 4.13$ (ddd, $\left.J_{1} 11.86 \mathrm{~Hz}, J_{2} 5.24 \mathrm{~Hz}, J_{3} 1.69 \mathrm{~Hz}, 1 \mathrm{H}\right), 4.36$ (dd, 
$\left.J_{1} 11.86 \mathrm{~Hz}, J_{2} 2.31 \mathrm{~Hz}, 1 \mathrm{H}\right), 7.23-7.37(\mathrm{~m}, 5 \mathrm{H}) ;{ }^{13} \mathrm{C} \mathrm{NMR}$ $\left(100 \mathrm{MHz}, \mathrm{CDCl}_{3}\right) \delta 25.3,40.1,48.4,66.0,69.2,77.7$, 125.9, 127.6, 128.5, 142.1. (CAS 63500-72-1).

Anti diastereoisomer: Eluent: hexane/ethyl acetate (1:1), Rf 0.81 ; colorless oil, yield 33\%; ${ }^{1} \mathrm{HNMR}\left(400 \mathrm{MHz}, \mathrm{CDCl}_{3}\right.$ ) $\delta 1.29(\mathrm{~s}, 3 \mathrm{H}), 1.49-1.56(\mathrm{~m}, 2 \mathrm{H}), 1.64\left(\mathrm{dd}, J_{1} 13.71 \mathrm{~Hz}\right.$, $\left.J_{2} 11.71 \mathrm{~Hz}, 1 \mathrm{H}\right), 1.73-1.83(\mathrm{~m}, 2 \mathrm{H}), 3.90-4.03(\mathrm{~m}, 2 \mathrm{H})$, $4.70\left(\mathrm{dd}, J_{1} 11.71 \mathrm{~Hz}, J_{2} 2.31 \mathrm{~Hz}, 1 \mathrm{H}\right), 7.21-7.43(\mathrm{~m}, 5 \mathrm{H})$; ${ }^{13} \mathrm{C} \mathrm{NMR}\left(100 \mathrm{MHz}, \mathrm{CDCl}_{3}\right) \delta 31.8,38.5,46.6,64.1,68.1$, 75.2, 125.9, 127.4, 128.4, 142.8. (CAS 63500-72-1).

\section{(E)-4-Methyl-2-styryltetrahydro-2H-pyran-4-ol (7a)}

Syn diastereoisomer: Eluent: hexane/ethyl acetate (1:1), Rf 0.56; colorless oil, yield 58\%; IR $v_{\max } / \mathrm{cm}^{-1} 3431,2969$, 2935, 2851, 1713, 1599, 1494, 1449, 1377, 1250, 1174, 1104, 965, 935, 880, 818, 753, 738, 693 (film); ${ }^{1} \mathrm{H}$ NMR $\left(400 \mathrm{MHz}, \mathrm{CDCl}_{3}\right) \delta 1.39(\mathrm{~s}, 3 \mathrm{H}), 1.60(\mathrm{t}, J 12.87 \mathrm{~Hz}, 1 \mathrm{H})$, $1.64\left(\mathrm{dq}, J_{1} 12.87 \mathrm{~Hz}, J_{2} 2.22 \mathrm{~Hz}, 1 \mathrm{H}\right), 1.77\left(\mathrm{td}, J_{1} 12.87 \mathrm{~Hz}\right.$, $\left.J_{2} 5.08 \mathrm{~Hz}, 1 \mathrm{H}\right), 1.81\left(\mathrm{dt}, J_{1} 12.87 \mathrm{~Hz}, J_{2} 2.22 \mathrm{~Hz}, 1 \mathrm{H}\right), 2.05$ (s, OH), 3.55 (td, $\left.J_{1} 12.23 \mathrm{~Hz}, J_{2} 2.38 \mathrm{~Hz}, 1 \mathrm{H}\right), 4.02(\mathrm{ddq}$, $J_{1} 12.23 \mathrm{~Hz}, J_{2} 5.88 \mathrm{~Hz}, J_{3} 1.43 \mathrm{~Hz}, 1 \mathrm{H}$ ), 4.06 (ddd, $\left.J_{1} 12.23 \mathrm{~Hz}, J_{2} 5.08 \mathrm{~Hz}, J_{3} 2.22 \mathrm{~Hz}, 1 \mathrm{H}\right), 6.19\left(\mathrm{dd}, J_{1}\right.$ $\left.16.05 \mathrm{~Hz}, J_{2} 5.88 \mathrm{~Hz}, 1 \mathrm{H}\right), 6.61\left(\mathrm{dd}, J_{1} 16.05 \mathrm{~Hz}, J_{2} 1.43 \mathrm{~Hz}\right.$, $1 \mathrm{H}), 7.20-7.39(\mathrm{~m}, 5 \mathrm{H}) ;{ }^{13} \mathrm{C} \mathrm{NMR}\left(100 \mathrm{MHz}, \mathrm{CDCl}_{3}\right)$ $\delta$ 25.4, 40.3, 46.5, 65.3, 68.8, 75.8, 126.5, 127.7, 128.6, 129.7, 130.5, 136.7; MS: $\mathrm{m} / \mathrm{z} 218\left(\mathrm{M}^{+}, 34 \%\right), 204$ (2), 200 (38), 185 (57), 171 (2), 148 (19), 147 (100), 129 (65), 115 (57), 104 (44), 91 (57), 71 (72), 55 (27); HRMS [M- $\left.\mathrm{Na}^{+}\right]$ Found: 241.1199. Calc. for $\mathrm{C}_{14} \mathrm{H}_{18} \mathrm{O}_{2}: 241.1204$.

Anti diastereoisomer: Eluent: hexane/ethyl acetate (1:1), Rf 0.73; colorless oil, yield 33\%; IR $v_{\max } / \mathrm{cm}^{-1} 3430$, 2943, 2919, 2855, 1674, 1603, 1518, 1458, 1381, 1281, 1124, 1088, 1035, 935, 886, 818, 741, 637 (film); ${ }^{1} \mathrm{H}$ NMR $\left(400 \mathrm{MHz}, \mathrm{CDCl}_{3}\right) \delta 1.29(\mathrm{~s}, 3 \mathrm{H}), 1.49\left(\mathrm{dq}, J_{1} 12.94 \mathrm{~Hz}\right.$, $\left.J_{2} 2.31 \mathrm{~Hz}, 1 \mathrm{H}\right), 1.51\left(\mathrm{dd}, J_{1} 13.56 \mathrm{~Hz}, J_{2} 11.51 \mathrm{~Hz}, 1 \mathrm{H}\right)$, $1.66-1.76(\mathrm{~m}, 2 \mathrm{H}), 1.89(\mathrm{~s}, \mathrm{OH}), 3.89\left(\mathrm{td}, J_{1} 11.40 \mathrm{~Hz}\right.$, $\left.J_{2} 2.15 \mathrm{~Hz}, 1 \mathrm{H}\right), 3.90\left(\mathrm{dd}, J_{1} 7.24 \mathrm{~Hz}, J_{2} 1.85 \mathrm{~Hz}, 1 \mathrm{H}\right), 4.35$ (ddq, $\left.J_{1} 11.40 \mathrm{~Hz}, J_{2} 6.01 \mathrm{~Hz}, J_{3} 1.54 \mathrm{~Hz}, 1 \mathrm{H}\right), 6.17$ (dd, $\left.J_{1} 16.02 \mathrm{~Hz}, J_{2} 6.01 \mathrm{~Hz}, 1 \mathrm{H}\right), 6.61\left(\mathrm{dd}, J_{1} 16.02 \mathrm{~Hz}\right.$, $\left.J_{2} 1.54 \mathrm{~Hz}, 1 \mathrm{H}\right), 7.18-7.40(\mathrm{~m}, 5 \mathrm{H}) ;{ }^{13} \mathrm{C}$ NMR $(100 \mathrm{MHz}$, $\left.\mathrm{CDCl}_{3}\right) \delta 31.7,38.3,44.7,63.5,67.7,73.4,126.3,127.4$, 128.4, 130.1, 130.2, 136.9; MS: $\mathrm{m} / \mathrm{z} 218\left(\mathrm{M}^{+}, 20 \%\right), 207$ (3), 200 (24), 185 (42), 171 (3), 148 (19), 147 (100), 129 (85), 115 (60), 104 (43), 91 (63), 71 (82), 55 (49); HRMS [M-Na+ ${ }^{+}$Found: 241.1200. Calc. for $\mathrm{C}_{14} \mathrm{H}_{18} \mathrm{O}_{2}: 241.1204$.

2-(4-Hydroxyphenyl)-4-methyltetrahydro-2H-pyran-4-ol (7b)

Syn diastereoisomer: Eluent: hexane/ethyl acetate (1:1), Rf 0.22; colorless oil, yield 13\%; IR $v_{\max } / \mathrm{cm}^{-1} 3363,2940$, 2861, 1614, 1518, 1443, 1377, 1250, 1225, 1170, 1143,
1080, 1047, 1013, 939, 899, 506 (film); ${ }^{1} \mathrm{H}$ NMR (400 MHz, $\left.\mathrm{CDCl}_{3}\right) \delta 1.39(\mathrm{~s}, 3 \mathrm{H}), 1.56-1.86(\mathrm{~m}, 4 \mathrm{H}), 3.63\left(\mathrm{td}, J_{1} 12.07\right.$ $\left.\mathrm{Hz}, J_{2} 2.25 \mathrm{~Hz}, 1 \mathrm{H}\right), 4.01$ (ddd, $J_{1} 12.07 \mathrm{~Hz}, J_{2} 5.30 \mathrm{~Hz}$, $\left.J_{3} 2.25 \mathrm{~Hz}, 1 \mathrm{H}\right), 4.31\left(\mathrm{dd}, J_{1} 12.07 \mathrm{~Hz}, J_{2} 2.25 \mathrm{~Hz}, 1 \mathrm{H}\right)$, $6.74\left(\mathrm{dt}, J_{1} 8.59 \mathrm{~Hz}, J_{2} 1.95 \mathrm{~Hz}, 2 \mathrm{H}\right), 7.16\left(\mathrm{dt}, J_{1} 8.59 \mathrm{~Hz}\right.$, $\left.J_{2} 1.95 \mathrm{~Hz}, 2 \mathrm{H}\right) ;{ }^{13} \mathrm{C} \mathrm{NMR}\left(100 \mathrm{MHz}, \mathrm{CDCl}_{3}\right) \delta 25.2,41.1$, 49.1, 66.8, 69.5, 78.8, 116.1, 128.5, 134.6, 158.0; MS: m/z $208\left(\mathrm{M}^{+}, 2 \%\right), 190$ (8), 175 (29), 161 (1), 137 (3), 121 (70), 119 (28), 91 (32), 77 (38), 71 (75), 58 (100); HRMS [M-Na+] Found: 231.0992. Calc. for $\mathrm{C}_{12} \mathrm{H}_{16} \mathrm{O}_{3}: 231.0997$.

Anti diastereoisomer: Eluent: hexane/ethyl acetate (1:1), Rf 0.49; white solid, yield 54\%, m.p. $194{ }^{\circ} \mathrm{C}$; IR v $v_{\max } / \mathrm{cm}^{-1}$ 3467, 2920, 2899, 2855, 1671, 1615, 1518, 1466, 1373, 1261, 1084, 1039, 1007, 902, 830, 737 (KBr); ${ }^{1} \mathrm{H}$ NMR $\left(400 \mathrm{MHz}, \mathrm{CDCl}_{3}\right) \delta 1.25(\mathrm{~s}, 3 \mathrm{H}), 1.52\left(\mathrm{dq}, J_{1} 13.84 \mathrm{~Hz}\right.$, $\left.J_{2} 1.69 \mathrm{~Hz}, 1 \mathrm{H}\right), 1.62\left(\mathrm{dd}, J_{1} 13.70 \mathrm{~Hz}, J_{2} 11.30 \mathrm{~Hz}, 1 \mathrm{H}\right.$ ), $1.65-1.74(\mathrm{~m}, 3 \mathrm{H}), 3.86\left(\mathrm{ddd}, J_{1} 11.30 \mathrm{~Hz}, J_{2} 5.22 \mathrm{~Hz}\right.$, $\left.J_{3} 1.13 \mathrm{~Hz}, 1 \mathrm{H}\right), 3.94\left(\mathrm{td}, J_{1} 11.30 \mathrm{~Hz}, J_{2} 2.40 \mathrm{~Hz}, 1 \mathrm{H}\right), 4.61$ $\left(\mathrm{dd}, J_{1} 11.30 \mathrm{~Hz}, J_{2} 2.40 \mathrm{~Hz}, 1 \mathrm{H}\right), 4.86(\mathrm{~s}, \mathrm{OH}), 6.74\left(\mathrm{dt}, J_{1}\right.$ $\left.8.61 \mathrm{~Hz}, J_{2} 1.97 \mathrm{~Hz}, 2 \mathrm{H}\right), 7.16\left(\mathrm{dt}, J_{1} 8.61 \mathrm{~Hz}, J_{2} 1.97 \mathrm{~Hz}\right.$, $2 \mathrm{H}) ;{ }^{13} \mathrm{C}$ NMR (100 MHz, $\left.\mathrm{CDCl}_{3}\right) \delta 31.6,39.1,47.1,65.2$, 68.5, 76.6, 116.1, 128.6, 134.8, 157.9; MS: $\mathrm{m} / \mathrm{z} 208\left(\mathrm{M}^{+}\right.$, 11\%), 190 (36), 176 (12), 175 (100), 161 (2), 137 (5), 121 (81), 119 (22), 91 (19), 71 (37), 58 (43); HRMS [M- $\left.\mathrm{Na}^{+}\right]$ Found: 231.0991. Calc. for $\mathrm{C}_{12} \mathrm{H}_{16} \mathrm{O}_{3}: 231.0997$.

2-(4-Methoxyphenyl)-4-methyltetrahydro-2H-pyran-4-ol (7c)

Syn diastereoisomer: Eluent: hexane/ethyl acetate (2:1), Rf 0.31; colorless oil, yield 27\%; IR $v_{\max } / \mathrm{cm}^{-1} 3426,2948$, 2915, 2847, 1614, 1587, 1518, 1470, 1377, 1249, 1176, 1080, 1031, 942, 898, 830, 770, 737, 605 (film); ${ }^{1} \mathrm{H}$ NMR $\left(400 \mathrm{MHz}, \mathrm{CDCl}_{3}\right) \delta 1.43(\mathrm{~s}, 3 \mathrm{H}), 1.66\left(\mathrm{qd}, J_{1} 12.87 \mathrm{~Hz}\right.$, $\left.J_{2} 2.38 \mathrm{~Hz}, 1 \mathrm{H}\right), 1.70-1.88(\mathrm{~m}, 4 \mathrm{H}), 1.95(\mathrm{~s}, \mathrm{OH}), 3.61$ (td, $\left.J_{1} 12.87 \mathrm{~Hz}, J_{2} 1.74 \mathrm{~Hz}, 1 \mathrm{H}\right), 3.79$ (s, 3H), 4.10 (ddd, $\left.J_{1} 12.08 \mathrm{~Hz}, J_{2} 5.24 \mathrm{~Hz}, J_{3} 1.74 \mathrm{~Hz}, 1 \mathrm{H}\right), 4.30\left(\mathrm{dd}, J_{1} 12.08 \mathrm{~Hz}\right.$, $\left.J_{2} 1.74 \mathrm{~Hz}, 1 \mathrm{H}\right), 6.87\left(\mathrm{dt}, J_{1} 8.74 \mathrm{~Hz}, J_{2} 2.06 \mathrm{~Hz}, 2 \mathrm{H}\right), 7.27$ $\left(\mathrm{dt}, J_{1} 8.74 \mathrm{~Hz}, J_{2} 2.06 \mathrm{~Hz}, 2 \mathrm{H}\right) ;{ }^{13} \mathrm{C} \mathrm{NMR}\left(100 \mathrm{MHz}, \mathrm{CDCl}_{3}\right)$ $\delta$ 25.2, 40.3, 48.1, 55.2, 65.8, 69.1, 77.2, 113.7, 127.2, 134.2, 159.0; MS: $\mathrm{m} / \mathrm{z} 222\left(\mathrm{M}^{+}, 16 \%\right), 204(30), 190(13), 189(100)$, 173 (5), 151 (6), 135 (95), 133 (23), 119 (18), 92 (10), 91 (26), 71 (46), 58 (31); HRMS [M-Na+] Found: 245.1152. Calc. for $\mathrm{C}_{13} \mathrm{H}_{18} \mathrm{O}_{3}: 245.1154$.

Anti diastereoisomer: Eluent: hexane/ethyl acetate (2:1), Rf 0.46; white solid, yield $62 \%$, m.p. $82{ }^{\circ} \mathrm{C}$; IR $v_{\max } / \mathrm{cm}^{-1}$ 3422, 2947, 2919, 2843, 1611, 1518, 1462, 1377, 1249, 1176, 1144, 1084, 1035, 943, 898, 830, 766, 605 (film); ${ }^{1} \mathrm{H}$ NMR (400 MHz, $\left.\mathrm{CDCl}_{3}\right) \delta 1.27(\mathrm{~s}, 3 \mathrm{H}), 1.50(\mathrm{dq}$, $\left.J_{1} 13.82 \mathrm{~Hz}, J_{2} 2.22 \mathrm{~Hz}, 1 \mathrm{H}\right), 1.59-1.81(\mathrm{~m}, 4 \mathrm{H}), 3.78(\mathrm{~s}$, $3 \mathrm{H}), 3.92-3.98(\mathrm{~m}, 2 \mathrm{H}), 4.64\left(\mathrm{dd}, J_{1} 11.44 \mathrm{~Hz}, J_{2} 2.54 \mathrm{~Hz}\right.$, $1 \mathrm{H}), 6.86\left(\mathrm{dt}, J_{1} 8.74 \mathrm{~Hz}, J_{2} 2.06 \mathrm{~Hz}, 2 \mathrm{H}\right), 7.26(\mathrm{dt}$, 
$\left.J_{1} 8.74 \mathrm{~Hz}, J_{2} 2.06 \mathrm{~Hz}, 2 \mathrm{H}\right) ;{ }^{13} \mathrm{C} \mathrm{NMR}\left(100 \mathrm{MHz}, \mathrm{CDCl}_{3}\right)$ $\delta 31.8,38.4,46.4,55.3,64.1,68.1,74.9,113.9,127.5$, 135.0, 159.1; MS: m/z $222\left(\mathbf{M}^{+}, 15 \%\right), 204$ (29), 190 (13), 189 (100), 173 (4), 151 (5), 135 (86), 133 (23), 109 (15), 92 (9), 91 (21), 71 (37), 58 (27); HRMS [M-Na+] Found: 245.1156. Calc. for $\mathrm{C}_{13} \mathrm{H}_{18} \mathrm{O}_{3}: 245.1154$.

4-Methyl-2-(4-nitrophenyl)tetrahydro-2H-pyran-4-ol (7d) Syn diastereoisomer: Eluent: hexane/ethyl acetate (1:1), Rf 0.42; colorless oil, yield $38 \%$; IR $v_{\max } / \mathrm{cm}^{-1} 3418$, 2972, 2939, 2859, 1604, 1519, 1347, 1252, 1093, 1045, 1014, 940, 809, 738, 697, 595 (film); ${ }^{1} \mathrm{H}$ NMR (400 MHz, $\left.\mathrm{CDCl}_{3}\right) \delta 1.47(\mathrm{~s}, 3 \mathrm{H}), 1.63\left(\mathrm{t}, J_{1} 12.23 \mathrm{~Hz}, 1 \mathrm{H}\right), 1.71$ (dq, $\left.J_{1} 12.87 \mathrm{~Hz}, J_{2} 2.22 \mathrm{~Hz}, 1 \mathrm{H}\right), 1.82(\mathrm{~s}, \mathrm{OH}), 1.85$ $\left(\mathrm{td}, J_{1} 12.39 \mathrm{~Hz}, J_{2} 5.24 \mathrm{~Hz}, 1 \mathrm{H}\right), 1.91\left(\mathrm{dt}, J_{1} 12.87 \mathrm{~Hz}\right.$, $\left.J_{2} 2.22 \mathrm{~Hz}, 1 \mathrm{H}\right), 3.64\left(\mathrm{td}, J_{1} 12.87 \mathrm{~Hz}, J_{2} 2.22 \mathrm{~Hz}, 1 \mathrm{H}\right)$, 4.17 (ddd, $\left.J_{1} 12.39 \mathrm{~Hz}, J_{2} 5.24 \mathrm{~Hz}, J_{3} 2.22 \mathrm{~Hz}, 1 \mathrm{H}\right), 4.47$ $\left(\mathrm{dd}, J_{1} 12.23 \mathrm{~Hz}, J_{2} 2.22 \mathrm{~Hz}, 1 \mathrm{H}\right), 7.51\left(\mathrm{dt}, J_{1} 8.90 \mathrm{~Hz}\right.$, $\left.J_{2} 2.22 \mathrm{~Hz}, 2 \mathrm{H}\right), 8.19$ (dt, $J_{1} 8.90 \mathrm{~Hz}, J_{2} 2.22 \mathrm{~Hz}, 2 \mathrm{H}$ ); ${ }^{13} \mathrm{C}$ NMR $\left(100 \mathrm{MHz}, \mathrm{CDCl}_{3}\right) \delta 25.3,40.1,48.4,65.9$, 68.9, 76.5, 123.7, 126.5, 147.3, 149.6; MS: $m / 2.219\left(\mathrm{M}^{+}-18\right.$, 30\%), 204 (82), 188 (4), 174 (15), 152 (34), 135 (3), 120 (21), 107 (35), 103 (16), 91 (22), 71 (55), 58 (100); HRMS [M-Na+ $]$ Found: 260.0894. Calc. for $\mathrm{C}_{12} \mathrm{H}_{15} \mathrm{NO}_{4}: 260.0899$.

Anti diastereoisomer: Eluent: hexane/ethyl acetate (1:1), Rf 0.60; colorless oil, yield 32\%; IR $v_{\max } / \mathrm{cm}^{-1} 3474$, 2968, 2923, 2875, 1605, 1530, 1349, 1257, 1144, 1013, 985, 906, 803, 749, 697, 596 (film); ${ }^{1} \mathrm{H}$ NMR (400 MHz, $\left.\mathrm{CDCl}_{3}\right) \delta 1.32(\mathrm{~s}, 3 \mathrm{H}), 1.54\left(\mathrm{dd}, J_{1} 13.51 \mathrm{~Hz}, J_{2} 11.76 \mathrm{~Hz}\right.$, $1 \mathrm{H}), 1.58\left(\mathrm{dq}, J_{1} 13.51 \mathrm{~Hz}, J_{2} 2.22 \mathrm{~Hz}, 1 \mathrm{H}\right), 1.74(\mathrm{~s}, \mathrm{OH})$, $1.80\left(\mathrm{td}, J_{1} 13.66 \mathrm{~Hz}, J_{2} 6.51 \mathrm{~Hz}, 1 \mathrm{H}\right), 1.83\left(\mathrm{dt}, J_{1} 13.66 \mathrm{~Hz}\right.$, $\left.J_{2} 2.22 \mathrm{~Hz}, 1 \mathrm{H}\right), 3.99\left(\mathrm{td}, J_{1} 11.76 \mathrm{~Hz}, J_{2} 2.22 \mathrm{~Hz}, 1 \mathrm{H}\right)$, 4.03 (ddd, $J_{1} 11.76 \mathrm{~Hz}, J_{2} 6.51 \mathrm{~Hz}, J_{3} 2.22 \mathrm{~Hz}, 1 \mathrm{H}$ ), 4.84 $\left(\mathrm{dd}, J_{1} 11.76 \mathrm{~Hz}, J_{2} 2.22 \mathrm{~Hz}, 1 \mathrm{H}\right), 7.52\left(\mathrm{dt}, J_{1} 8.90 \mathrm{~Hz}\right.$, $\left.J_{2} 2.22 \mathrm{~Hz}, 2 \mathrm{H}\right), 8.18\left(\mathrm{dt}, J_{1} 8.90 \mathrm{~Hz}, J_{2} 2.22 \mathrm{~Hz}, 2 \mathrm{H}\right) ;{ }^{13} \mathrm{C}$ NMR $\left(100 \mathrm{MHz}, \mathrm{CDCl}_{3}\right) \delta 31.7,38.2,46.7,64.0,67.9$, 74.2, 123.5, 126.4, 147.0, 150.5; MS: $\mathrm{m} / \mathrm{z} 219\left(\mathrm{M}^{+}-18\right.$, 36\%), 204 (100), 202 (3), 188 (2), 174 (3), 152 (30), 144 (1), 120 (4), 103 (8), 91 (8), 71 (29), 58 (51); HRMS $\left[\mathrm{M}-\mathrm{Na}^{+}\right]$Found: 260.0901. Calc. for $\mathrm{C}_{12} \mathrm{H}_{15} \mathrm{NO}_{4}: 260.0899$.

\section{2-(3-Bromophenyl)-tetrahydro-4-methyl-2H-pyran-4-ol} (7e)

Syn diastereoisomer: Eluent: hexane/ethyl acetate (3:1), Rf 0.22; colorless oil, yield 41\%; IR $v_{\max } / \mathrm{cm}^{-1} 3422,2964$, 2939, 2855, 1716, 1595, 1569, 1474, 1337, 1252, 1092, 1045, 996, 942, 868, 822, 784, 747, 693, 598; ${ }^{1} \mathrm{H}$ NMR $\left(400 \mathrm{MHz}, \mathrm{CDCl}_{3}\right) \delta 1.41(\mathrm{~s}, 3 \mathrm{H}), 1.62\left(\mathrm{~d}, J_{1} 12.33 \mathrm{~Hz}, 1 \mathrm{H}\right)$, $1.65\left(\mathrm{dq}, J_{1} 12.79 \mathrm{~Hz}, J_{2} 2.46 \mathrm{~Hz}, 1 \mathrm{H}\right), 1.80\left(\mathrm{td}, J_{1} 12.79 \mathrm{~Hz}\right.$, $\left.J_{2} 5.24 \mathrm{~Hz}, 1 \mathrm{H}\right), 1.84\left(\mathrm{dt}, J_{1} 12.79, J_{2} 2.46 \mathrm{~Hz}, 1 \mathrm{H}\right), 1.23(\mathrm{~s}$, OH), $3.58\left(\mathrm{td}, J_{1} 12.33 \mathrm{~Hz}, J_{2} 2.31 \mathrm{~Hz}, 1 \mathrm{H}\right), 4.10$ (ddd, $J_{1}$ $\left.12.02 \mathrm{~Hz}, J_{2} 5.24 \mathrm{~Hz}, J_{3} 1.54 \mathrm{~Hz}, 1 \mathrm{H}\right), 4.31\left(\mathrm{dd}, J_{1} 12.02 \mathrm{~Hz}\right.$, $\left.J_{2} 1.54 \mathrm{~Hz}, 1 \mathrm{H}\right), 7.18\left(\mathrm{t}, J_{1} 7.70 \mathrm{~Hz}, 1 \mathrm{H}\right), 7.24\left(\mathrm{dt}, J_{1} 7.70 \mathrm{~Hz}\right.$, $\left.J_{2} 1.54 \mathrm{~Hz}, 1 \mathrm{H}\right), 7.38\left(\mathrm{dt}, J_{1} 7.70 \mathrm{~Hz}, J_{2} 1.54 \mathrm{~Hz}, 1 \mathrm{H}\right), 7.50$ $\left(\mathrm{t}, J_{1} 1.54 \mathrm{~Hz}, 1 \mathrm{H}\right) ;{ }^{13} \mathrm{C}$ NMR $\left(100 \mathrm{MHz}, \mathrm{CDCl}_{3}\right) \delta 25.2$, 40.1, 48.3, 65.8, 69.0, 76.8, 122.5, 124.4, 129.0, 129.9, 130.6, 144.4; MS: $m / z 272\left(\mathrm{M}^{+}+2,2 \%\right), 254$ (22), 237 (49), 227 (2), 209 (1), 199 (1), 185 (35), 173 (6), 157 (13), 145 (6), 128 (7), 115 (5), 103 (37), 78 (16), 71 (90), 58 (100); HRMS [M-Na ${ }^{+}$Found: 293.0146. Calc. for $\mathrm{C}_{12} \mathrm{H}_{15} \mathrm{BrO}_{2}$ : 293.0153.

Anti diastereoisomer: Eluent: hexane/ethyl acetate (3:1), Rf 0.28; colorless oil, yield 28\%; IR $v_{\max } / \mathrm{cm}^{-1} 3467,3067$, 2969, 2919, 2873, 1713, 1596, 1568, 1477, 1361, 1261, 1044, 984, 925, 864, 816, 783, 739, 692 (film); ${ }^{1} \mathrm{H}$ NMR $\left(400 \mathrm{MHz}, \mathrm{CDCl}_{3}\right) \delta 1.26(\mathrm{~s}, 3 \mathrm{H}), 1.49\left(\mathrm{dq}, J_{1} 13.84 \mathrm{~Hz}\right.$, $\left.J_{2} 1.29 \mathrm{~Hz}, 1 \mathrm{H}\right), 1.55\left(\mathrm{dd}, J_{1} 13.84 \mathrm{~Hz}, J_{2} 11.80 \mathrm{~Hz}, 1 \mathrm{H}\right)$, 1.68-1.78 (m, 2H), $1.84(\mathrm{~s}, \mathrm{OH}), 3.58\left(\mathrm{td}, J_{1} 11.53 \mathrm{~Hz}\right.$, $\left.J_{2} 2.14 \mathrm{~Hz}, 1 \mathrm{H}\right), 4.66$ (ddd, $J_{1} 11.53 \mathrm{~Hz}, J_{2} 6.24 \mathrm{~Hz}$, $\left.J_{3} 1.29 \mathrm{~Hz}, 1 \mathrm{H}\right), 4.31\left(\mathrm{dd}, J_{1} 11.53 \mathrm{~Hz}, J_{2} 2.14 \mathrm{~Hz}, 1 \mathrm{H}\right)$, $7.16\left(\mathrm{t}, J_{1} 7.76 \mathrm{~Hz}, 1 \mathrm{H}\right), 7.23\left(\mathrm{~d}, J_{1} 7.76 \mathrm{~Hz}, 1 \mathrm{H}\right), 7.36(\mathrm{dt}$, $\left.J_{1} 7.76 \mathrm{~Hz}, J_{2} 1.54 \mathrm{~Hz}, 1 \mathrm{H}\right), 7.51\left(\mathrm{t}, J_{1} 1.54 \mathrm{~Hz}, 1 \mathrm{H}\right) ;{ }^{13} \mathrm{CNMR}$ $\left(100 \mathrm{MHz}, \mathrm{CDCl}_{3}\right) \delta 31.6,38.3,46.4,64.0,67.8,74.4$, 122.4, 124.5, 128.9, 129.8, 130.3, 145.0; MS: $\mathrm{m} / \mathrm{z} 272$ $\left(\mathrm{M}^{+}+2,2 \%\right), 237$ (66), 209 (1), 200 (3), 185 (41), 173(7), 157 (15), 145 (6), 128 (6), 115 (5), 103 (31), 78 (16), 71 (82), 58 (100); HRMS [M-Na ${ }^{+}$Found: 293.0152. Calc. for $\mathrm{C}_{12} \mathrm{H}_{15} \mathrm{BrO}_{2}: 293.0153$.

\section{2-Cyclohexyl-4-methyltetrahydro-2H-pyran-4-ol (7f)}

Syn diastereoisomer: Eluent: hexane/ethyl acetate (3:1), Rf 0.62; white solid, yield $44 \%$, m.p. $90{ }^{\circ} \mathrm{C}$; IR $v_{\max } / \mathrm{cm}^{-1}$ 3414, 2920, 2855, 1708, 1450, 1381, 1333, 1216, 1172, 1146, 939, 862, 826, 741, 649 (KBr); ${ }^{1} \mathrm{H}$ NMR (400 MHz, $\left.\mathrm{CDCl}_{3}\right) \delta$ 0.72-2.04 (m, 15H), $1.31(\mathrm{~s}, 3 \mathrm{H}), 2.47(\mathrm{~s}, \mathrm{OH})$, $3.03\left(\mathrm{ddd}, J_{1} 11.27 \mathrm{~Hz}, J_{2} 6.18 \mathrm{~Hz}, J_{3} 2.09 \mathrm{~Hz}, 1 \mathrm{H}\right), 3.40$ (td, $J_{1} 11.86 \mathrm{~Hz}, J_{2} 2.89 \mathrm{~Hz}, 1 \mathrm{H}$ ), 3.96 (ddd, $J_{1} 11.86 \mathrm{~Hz}$, $\left.J_{2} 6.18 \mathrm{~Hz}, J_{3} 2.09 \mathrm{~Hz}, 1 \mathrm{H}\right) ;{ }^{13} \mathrm{C} \mathrm{NMR}\left(100 \mathrm{MHz}, \mathrm{CDCl}_{3}\right)$ $\delta$ 25.5, 26.2, 26.6, 28.7, 28.9, 29.0, 40.8, 43.0, 43.5, 65.5, 69.3, 79.8; MS: $m / z, 180\left(\mathrm{M}^{+}-18,2 \%\right), 135$ (1), $115(46)$, 95 (10), 81 (7), 71 (100), 55 (22); HRMS [M-Na+ Found: 221.1519. Calc. for $\mathrm{C}_{12} \mathrm{H}_{22} \mathrm{O}_{2}: 221.1517$.

Anti diastereoisomer: Eluent: hexane/ethyl acetate (3:1), Rf 0.40; colorless oil, yield 27\%; IR $v_{\max } / \mathrm{cm}^{-1} 3402$, 2919, 2843, 1703, 1478, 1450, 1398, 1096, 1007, 863, 826, 733, 645 (film); ${ }^{1} \mathrm{H}$ NMR (400 MHz, $\mathrm{CDCl}_{3}$ ) $\delta$ 0.91-1.98 (m, 15H), $1.26(\mathrm{~s}, 3 \mathrm{H}), 3.12$ (s, OH), 3.36 (ddd, $J_{1} 11.40 \mathrm{~Hz}$, $\left.J_{2} 6.31 \mathrm{~Hz}, J_{3} 1.54 \mathrm{~Hz}, 1 \mathrm{H}\right), 3.73\left(\mathrm{td}, J_{1} 11.40 \mathrm{~Hz}, J_{2} 1.54 \mathrm{~Hz}\right.$, $1 \mathrm{H}), 3.84\left(\mathrm{dd}, J_{1} 11.40 \mathrm{~Hz}, J_{2} 6.31 \mathrm{~Hz}, 1 \mathrm{H}\right) ;{ }^{13} \mathrm{C} \mathrm{NMR}$ $\left(100 \mathrm{MHz}, \mathrm{CDCl}_{3}\right) \delta 26.1,26.2,26.5,28.5,28.9,31.9$, 38.8, 41.4, 42.4, 63.7, 67.9, 77.1; MS: $m / z 180\left(\mathrm{M}^{+}-18\right.$, 11\%), 165 (3), 135 (2), 125 (6), 115 (78), 95 (28), 83 (17), 
69 (100), 55 (41); HRMS [M-Na+] Found: 221.1513. Calc. for $\mathrm{C}_{12} \mathrm{H}_{22} \mathrm{O}_{2}: 221.1517$.

(E)-4-Methyl-2-(prop-1-enyl)tetrahydro-2H-pyran-4-ol (7g)

Syn diastereoisomer: Eluent: hexane/ethyl acetate (2:1), Rf 0.60; colorless oil, yield 25\%; ${ }^{1} \mathrm{H}$ NMR (400 MHz, $\left.\mathrm{CDCl}_{3}\right) \delta 1.34(\mathrm{~s}, 3 \mathrm{H}), 1.43-1.62(\mathrm{~m}, 5 \mathrm{H}), 1.70(\mathrm{dq}$, $\left.J_{1} 6.55 \mathrm{~Hz}, J_{2} 0.80 \mathrm{~Hz}, 3 \mathrm{H}\right), 3.48\left(\mathrm{td}, J_{1} 12.19 \mathrm{~Hz}, J_{2} 2.46 \mathrm{~Hz}\right.$, $1 \mathrm{H}), 3.78$ (ddq, $J_{1} 11.33 \mathrm{~Hz}, J_{2} 6.38 \mathrm{~Hz}, J_{3} 1.06 \mathrm{~Hz}, 1 \mathrm{H}$ ), 3.99 (ddd, $\left.J_{1} 11.95 \mathrm{~Hz}, J_{2} 5.11 \mathrm{~Hz}, J_{3} 1.99 \mathrm{~Hz}, 1 \mathrm{H}\right), 5.49$ (ddq, $\left.J_{1} 15.38 \mathrm{~Hz}, J_{2} 6.43 \mathrm{~Hz}, J_{3} 1.63 \mathrm{~Hz}, 1 \mathrm{H}\right), 5.72$ (ddq, $\left.J_{1} 15.38 \mathrm{~Hz}, J_{2} 6.49 \mathrm{~Hz}, J_{3} 1.05 \mathrm{~Hz}, 1 \mathrm{H}\right) ;{ }^{13} \mathrm{C}$ NMR $\left(100 \mathrm{MHz}, \mathrm{CDCl}_{3}\right) \delta 17.9,27.7,39.7,46.4,58.4,73.2$, 75.1, 128.0, 133.9; (CAS 69359-03-1).

Anti diastereoisomer: Eluent: hexane/ethyl acetate (2:1), Rf 0.73; colorless oil, yield 37\%; ${ }^{1} \mathrm{H}$ NMR $\left(400 \mathrm{MHz}, \mathrm{CDCl}_{3}\right) \delta 1.27(\mathrm{~s}, 3 \mathrm{H}), 1.40-1.60(\mathrm{~m}, 5 \mathrm{H})$, $1.69\left(\mathrm{dq}, J_{1} 6.46 \mathrm{~Hz}, J_{2} 0.84 \mathrm{~Hz}, 3 \mathrm{H}\right), 3.82\left(\mathrm{td}, J_{1} 11.50 \mathrm{~Hz}\right.$, $\left.J_{2} 2.28 \mathrm{~Hz}, 1 \mathrm{H}\right), 3.85\left(\mathrm{dd}, J_{1} 6.35 \mathrm{~Hz}, J_{2} 1.46 \mathrm{~Hz}, 1 \mathrm{H}\right), 4.10$ (ddq, $\left.J_{1} 11.50 \mathrm{~Hz}, J_{2} 6.46 \mathrm{~Hz}, J_{3} 0.84 \mathrm{~Hz}, 1 \mathrm{H}\right), 5.46$ (ddq, $\left.J_{1} 15.35 \mathrm{~Hz}, J_{2} 6.56 \mathrm{~Hz}, J_{3} 1.46 \mathrm{~Hz}, 1 \mathrm{H}\right), 5.72$ (ddq, $\left.J_{1} 15.35 \mathrm{~Hz}, J_{2} 6.48 \mathrm{~Hz}, J_{3} 1.09 \mathrm{~Hz}, 1 \mathrm{H}\right) ;{ }^{13} \mathrm{C}$ NMR $\left(100 \mathrm{MHz}, \mathrm{CDCl}_{3}\right) \delta 17.8,31.8,38.4,44.7,63.5,67.8$, 73.5, 127.2, 131.9; (CAS 69359-03-1).

(E)-2-(But-1-enyl)-4-methyltetrahydro-2H-pyran-4-ol (7h)

Syn diastereoisomer: Eluent: hexane/ethyl acetate (3:1), Rf 0.28; colorless oil, yield 41\%; IR $v_{\max } / \mathrm{cm}^{-1} 3418$, 2964, 2931, 2859, 1675, 1462, 1381, 1337, 1253, 1081, 971, 830 (film); ${ }^{1} \mathrm{H}$ NMR (400 MHz, $\left.\mathrm{CDCl}_{3}\right) \delta 1.00$ (t, $\left.J_{1} 7.49 \mathrm{~Hz}, 3 \mathrm{H}\right), 1.35(\mathrm{~s}, 3 \mathrm{H}), 1.51\left(\mathrm{t}, J_{1} 11.83 \mathrm{~Hz}, 1 \mathrm{H}\right), 1.60$ (dq, $\left.J_{1} 12.89 \mathrm{~Hz}, J_{2} 2.23 \mathrm{~Hz}, 1 \mathrm{H}\right), 1.70\left(\mathrm{dt}, J_{1} 12.76 \mathrm{~Hz}\right.$, $\left.J_{2} 2.36 \mathrm{~Hz}, 2 \mathrm{H}\right), 2.05\left(\mathrm{qt}, J_{1} 7.36 \mathrm{~Hz}, 2 \mathrm{H}\right), 3.49(\mathrm{td}$, $\left.J_{1} 12.10 \mathrm{~Hz}, J_{2} 2.49 \mathrm{~Hz}, 1 \mathrm{H}\right), 3.80\left(\mathrm{ddq}, J_{1} 11.18 \mathrm{~Hz}\right.$, $\left.J_{2} 6.31 \mathrm{~Hz}, J_{3} 1.18 \mathrm{~Hz}, 1 \mathrm{H}\right), 4.00\left(\mathrm{ddd}, J_{1} 11.83 \mathrm{~Hz}\right.$, $\left.J_{2} 5.13 \mathrm{~Hz}, J_{3} 1.84 \mathrm{~Hz}, 1 \mathrm{H}\right), 5.46\left(\mathrm{ddt}, J_{1} 15.52 \mathrm{~Hz}\right.$, $\left.J_{2} 6.31 \mathrm{~Hz}, J_{3} 1.57 \mathrm{~Hz}, 1 \mathrm{H}\right), 5.75\left(\mathrm{dtd}, J_{1} 15.52 \mathrm{~Hz}\right.$, $\left.J_{2} 6.18 \mathrm{~Hz}, J_{3} 1.05 \mathrm{~Hz}, 1 \mathrm{H}\right) ;{ }^{13} \mathrm{C} \mathrm{NMR}\left(100 \mathrm{MHz}, \mathrm{CDCl}_{3}\right)$ $\delta$ 13.3, 25.3, 25.4, 40.4, 46.6, 65.3, 68.9, 76.0, 129.3, 134.4; MS: $m / z 170\left(\mathrm{M}^{+}, 1 \%\right), 155$ (4), 141 (25), 123 (3), 107 (2), 99 (4), 83 (10), 71 (100), 55 (18); HRMS [M-Na+ Found: 193.1204. Calc. for $\mathrm{C}_{10} \mathrm{H}_{18} \mathrm{O}_{2}: 193.1204$.

Anti diastereoisomer: Eluent: hexane/ethyl acetate (3:1), Rf 0.20; colorless oil, yield 17\%; IR $v_{\max } / \mathrm{cm}^{-1} 3434$, 2964, 2927, 2875, 1623, 1466, 1381, 1264, 1080, 971, 908, 735, 650 (film); ${ }^{1} \mathrm{H}$ NMR (400 MHz, $\left.\mathrm{CDCl}_{3}\right) \delta 0.99$ (t, $\left.J_{1} 7.39 \mathrm{~Hz}, 3 \mathrm{H}\right), 1.27(\mathrm{~s}, 3 \mathrm{H}), 1.45\left(\mathrm{dd}, J_{1} 13.71 \mathrm{~Hz}\right.$, $\left.J_{2} 11.40 \mathrm{~Hz}, 1 \mathrm{H}\right), 1.59\left(\mathrm{dt}, J_{1} 13.71 \mathrm{~Hz}, J_{2} 2.46 \mathrm{~Hz}, 2 \mathrm{H}\right), 1.68$ (dq, $\left.J_{1} 13.87 \mathrm{~Hz}, J_{2} 6.16 \mathrm{~Hz}, 1 \mathrm{H}\right), 2.05\left(\mathrm{qt}, J_{1} 7.24 \mathrm{~Hz}, 2 \mathrm{H}\right)$, $3.83\left(\mathrm{td}, J_{1} 11.56 \mathrm{~Hz}, J_{2} 2.31,1 \mathrm{H}\right), 3.84-3.90(\mathrm{~m}, 1 \mathrm{H}), 4.12$ (ddd, $\left.J_{1} 10.48 \mathrm{~Hz}, J_{2} 6.62 \mathrm{~Hz}, J_{3} 1.38 \mathrm{~Hz}, 1 \mathrm{H}\right), 5.44$ (ddt, $\left.J_{1} 15.41 \mathrm{~Hz}, J_{2} 6.47 \mathrm{~Hz}, J_{3} 1.54 \mathrm{~Hz}, 1 \mathrm{H}\right), 5.76(\mathrm{dtd}$, $\left.J_{1} 15.56 \mathrm{~Hz}, J_{2} 6.31 \mathrm{~Hz}, J_{3} 1.07 \mathrm{~Hz}, 1 \mathrm{H}\right) ;{ }^{13} \mathrm{CNMR}(100 \mathrm{MHz}$, $\left.\mathrm{CDCl}_{3}\right) \delta 13.3,25.3,31.8,38.4,44.8,63.5,67.9,73.6,129.6$, 134.1; MS: $m / z 170\left(\mathrm{M}^{+}, 2 \%\right), 152$ (3), 141 (22), 123 (6), 113 (2), 99 (3), 83 (13), 71 (100), 55 (21); HRMS [M- $\left.\mathrm{Na}^{+}\right]$ Found: 193.1202. Calc. for $\mathrm{C}_{10} \mathrm{H}_{18} \mathrm{O}_{2}: 193.1204$.

\section{4-Methyl-2-propyltetrahydro-2H-pyran-4-ol (10)}

Syn diastereoisomer: Eluent: hexane/ethyl acetate (3:1), Rf 0.28; colorless oil, yield 21\%; ${ }^{1} \mathrm{H}$ NMR $(400 \mathrm{MHz}$, $\left.\mathrm{CDCl}_{3}\right) \delta 0.91\left(\mathrm{t}, J_{1} 6.88 \mathrm{~Hz}, 3 \mathrm{H}\right), 1.32(\mathrm{~s}, 3 \mathrm{H}), 1.34-1.88(\mathrm{~m}$, 9H), 3.21-3.34 (m, 1H), $3.42\left(\mathrm{td}, J_{1} 11.96 \mathrm{~Hz}, J_{2} 2.99 \mathrm{~Hz}\right.$, 1H), 3.96 (ddd, $J_{1} 11.96 \mathrm{~Hz}, J_{2} 4.98 \mathrm{~Hz}, J_{3} 2.99 \mathrm{~Hz}, 1 \mathrm{H}$ ); ${ }^{13} \mathrm{C} \mathrm{NMR}\left(100 \mathrm{MHz}, \mathrm{CDCl}_{3}\right) \delta 14.0,18.7,25.4,38.4,40.7$, 46.6, 65.4, 68.9, 75.2; (CAS 723340-91-8).

Anti diastereoisomer: Eluent: hexane/ethyl acetate (3:1), Rf 0.46; colorless oil, yield 40\%; ${ }^{1} \mathrm{H}$ NMR $(400 \mathrm{MHz}$, $\left.\mathrm{CDCl}_{3}\right) \delta 0.91\left(\mathrm{t}, J_{1} 6.98 \mathrm{~Hz}, 3 \mathrm{H}\right), 1.25(\mathrm{~s}, 3 \mathrm{H}), 1.27-1.76$ $(\mathrm{m}, 8 \mathrm{H}), 1.95(\mathrm{~s}, \mathrm{OH}), 3.53-3.89(\mathrm{~m}, 3 \mathrm{H}) ;{ }^{13} \mathrm{C}$ NMR $\left(100 \mathrm{MHz}, \mathrm{CDCl}_{3}\right) \delta 14.0,18.5,31.7,38.2$, 38.6, 44.6, 63.5, 67.7, 72.6; (CAS 723340-91-8).

\section{2-Isobutyl-4-methyltetrahydro-2H-pyran-4-ol (11)-Florol ${ }^{\circledR}$}

Syn diastereoisomer: Eluent: hexane/ethyl acetate (3:1), Rf 0.48; colorless oil, yield 35\%; ${ }^{1} \mathrm{H}$ NMR (400 MHz, $\left.\mathrm{CDCl}_{3}\right) \delta 0.90\left(\mathrm{~d}, J_{1} 6.54 \mathrm{~Hz}, 6 \mathrm{H}\right), 1.12\left(\mathrm{ddd}, J_{1} 13.66 \mathrm{~Hz}\right.$, $\left.J_{2} 8.39 \mathrm{~Hz}, J_{3} 4.55,1 \mathrm{H}\right), 1.25(\mathrm{~s}, 3 \mathrm{H}), 1.28\left(\mathrm{dd}, J_{1} 13.66 \mathrm{~Hz}\right.$, $\left.J_{2} 11.52 \mathrm{~Hz}, 1 \mathrm{H}\right), 1.40-1.50(\mathrm{~m}, 3 \mathrm{H}), 1.53\left(\mathrm{dtl}, J_{1} 13.66 \mathrm{~Hz}\right.$, $\left.J_{2} 2.13 \mathrm{~Hz}, 1 \mathrm{H}\right), 1.63\left(\mathrm{td}, J_{1} 13.66 \mathrm{~Hz}, J_{2} 5.83,1 \mathrm{H}\right), 1.78$ (hept, $\left.J_{1} 6.54 \mathrm{~Hz}, 1 \mathrm{H}\right), 3.19$ (sl, OH), 3.65-3.84 (m, 2H), 3.76 (td, $\left.J_{1} 12.09 \mathrm{~Hz}, J_{2} 2.13 \mathrm{~Hz}, 1 \mathrm{H}\right) ;{ }^{13} \mathrm{C}$ NMR $(100 \mathrm{MHz}$, $\left.\mathrm{CDCl}_{3}\right) \delta 22.4,23.3,24.3,31.7,38.7,45.1,45.4,63.6,67.8$, 71.2; (CAS 723440-93-0).

Anti diastereoisomer: Eluent: hexane/ethyl acetate (3:1), Rf 0.22; colorless oil, yield 52\%; ${ }^{1} \mathrm{H}$ NMR $(400 \mathrm{MHz}$, $\left.\mathrm{CDCl}_{3}\right) \delta 0.90\left(\mathrm{~d}, J_{1} 6.54 \mathrm{~Hz}, 6 \mathrm{H}\right), 1.17\left(\mathrm{ddd}, J_{1} 12.66 \mathrm{~Hz}\right.$, $\left.J_{2} 8.25 \mathrm{~Hz}, J_{3} 4.55 \mathrm{~Hz}, 1 \mathrm{H}\right), 1.32(\mathrm{~s}, 3 \mathrm{H}), 1.37$ (t, $J_{1} 12.24 \mathrm{~Hz}$, $1 \mathrm{H}), 1.49$ (ddd, $J_{1} 12.66 \mathrm{~Hz}, J_{2} 8.25 \mathrm{~Hz}, J_{3} 5.97 \mathrm{~Hz}, 1 \mathrm{H}$ ), $1.58\left(\mathrm{dq}, J_{1} 12.66 \mathrm{~Hz}, J_{2} 2.13 \mathrm{~Hz}, 1 \mathrm{H}\right), 1.63\left(\mathrm{dt}, J_{1} 12.66 \mathrm{~Hz}\right.$, $\left.J_{2} 2.13 \mathrm{~Hz}, 1 \mathrm{H}\right), 1.71\left(\mathrm{td}, J_{1} 12.66 \mathrm{~Hz}, J_{2} 5.12 \mathrm{~Hz}, 1 \mathrm{H}\right), 1.77$ (hept, $\left.J_{1} 6.54 \mathrm{~Hz}, J_{2} 1.56 \mathrm{~Hz}, 1 \mathrm{H}\right), 2.96$ (s, OH), 3.32-3.37 (m, $1 \mathrm{H}), 3.41$ (td, $\left.J_{1} 12.24 \mathrm{~Hz}, J_{2} 2.13 \mathrm{~Hz}, 1 \mathrm{H}\right), 3.95$ (ddd, $\left.J_{1} 12.66 \mathrm{~Hz}, J_{2} 5.12 \mathrm{~Hz}, J_{3} 1.56 \mathrm{~Hz}, 1 \mathrm{H}\right) ;{ }^{13} \mathrm{CNMR}(100 \mathrm{MHz}$, $\left.\mathrm{CDCl}_{3}\right) \delta 22.3,23.1,24.3,25.3,40.5,45.4,46.9,65.3,68.8$, 73.6; (CAS 723440-93-0).

4-Methyl-2-propyltetrahydro-2H-pyran-4-yl acetate (12)-Clarycet ${ }^{\oplus}$

To a stirred solution of 4-methyl-2-propyltetrahydro$2 \mathrm{H}$-pyran-4-ol (10) $(500 \mathrm{mg}, 3.16 \mathrm{mmol})$ in pyridine $(3 \mathrm{~mL})$ 
are added acetic anhydride (322 mg, $3.16 \mathrm{mmol}, 1.0$ equiv). The mixture is magnetically stirred at room temperature for $7 \mathrm{~h}$. After completion of acetylation, the solution is neutralized with solid $\mathrm{NaHCO}_{3}$, filtered and solvent evaporated. Purification by flash column chromatography on silica gel, using a mixture of hexane/ethyl acetate (10:1) as eluent, afforded $180 \mathrm{mg}$ (90\%) of the title compound as colourless oil.

Syn diastereoisomer: Colorless oil, yield 90\%; ${ }^{1} \mathrm{H}$ NMR $\left(400 \mathrm{MHz}, \mathrm{CDCl}_{3}\right) \delta 0.91\left(\mathrm{t}, J_{1} 7.20 \mathrm{~Hz}, 3 \mathrm{H}\right), 1.54$ (ddq, $\left.J_{1} 12.60 \mathrm{~Hz}, J_{2} 11.50 \mathrm{~Hz}, J_{3} 0.90 \mathrm{~Hz}, 1 \mathrm{H}\right), 1.30-1.55(\mathrm{~m}$, $4 \mathrm{H}), 1.62\left(\mathrm{t}, J_{1} 0.90 \mathrm{~Hz}, 3 \mathrm{H}\right), 1.86$ (dddq, $J_{1} 12.80 \mathrm{~Hz}$, $\left.J_{2} 12.60 \mathrm{~Hz}, J_{3} 5.30 \mathrm{~Hz}, J_{4} 0.90 \mathrm{~Hz}, 1 \mathrm{H}\right), 1.97(\mathrm{~s}, 3 \mathrm{H}), 2.03$ (dddd, $J_{1} 12.80 \mathrm{~Hz}, J_{2} 2.30 \mathrm{~Hz}, J_{3} 2.20 \mathrm{~Hz}, J_{4} 1.90 \mathrm{~Hz}$, $1 \mathrm{H}), 2.10\left(\mathrm{dt}, J_{1} 12.60 \mathrm{~Hz}, J_{2} 2.20 \mathrm{~Hz}, 1 \mathrm{H}\right), 3.33(\mathrm{~m}, 1 \mathrm{H})$, 3.46 (ddd, $J_{1} 12.60 \mathrm{~Hz}, J_{2} 12.10 \mathrm{~Hz}, J_{3} 2.30 \mathrm{~Hz}, 1 \mathrm{H}$ ), 3.93 (ddd, $\left.J_{1} 12.10 \mathrm{~Hz}, J_{2} 5.30 \mathrm{~Hz}, J_{3} 1.90 \mathrm{~Hz}, 1 \mathrm{H}\right) ;{ }^{13} \mathrm{C}$ NMR $\left(100 \mathrm{MHz}, \mathrm{CDCl}_{3}\right) \delta 14.4,19.1,21.4,24.4,36.3,37.8,42.1$, 58.5, 66.7, 73.9, 170.2; (CAS 723340-91-8).

Anti diastereoisomer: Colorless oil, yield 90\%; ${ }^{1} \mathrm{H} \mathrm{NMR}\left(400 \mathrm{MHz}, \mathrm{CDCl}_{3}\right) \delta 0.89\left(\mathrm{t}, J_{1} 7.20 \mathrm{~Hz}, 3 \mathrm{H}\right)$, $1.02\left(\mathrm{dd}, J_{1} 13.80 \mathrm{~Hz}, J_{2} 11.30 \mathrm{~Hz}, 1 \mathrm{H}\right), 1.28$ (dd, $\left.J_{1} 14.10 \mathrm{~Hz}, J_{2} 12.60 \mathrm{~Hz}, J_{3} 5.30 \mathrm{~Hz}, 1 \mathrm{H}\right), 1.25-1.60(\mathrm{~m}$, $4 \mathrm{H}), 1.68$ (s, 3H), 2.05 (dddd, $J_{1} 14.10 \mathrm{~Hz}, J_{2} 2.30 \mathrm{~Hz}$, $\left.J_{3} 2.10 \mathrm{~Hz}, J_{4} 1.60 \mathrm{~Hz}, 1 \mathrm{H}\right), 2.20\left(\mathrm{dt}, J_{1} 13.80 \mathrm{~Hz}\right.$, $\left.J_{2} 2.30 \mathrm{~Hz}, 1 \mathrm{H}\right), 3.52$ (dddd, $J_{1} 11.40 \mathrm{~Hz}, J_{2} 7.40 \mathrm{~Hz}$, $\left.J_{3} 4.60 \mathrm{~Hz}, J_{4} 2.30 \mathrm{~Hz}, 1 \mathrm{H}\right), 3.57\left(\mathrm{ddd}, J_{1} 12.60 \mathrm{~Hz}\right.$, $\left.J_{2} 11.60 \mathrm{~Hz}, J_{3} 2.10 \mathrm{~Hz}, 1 \mathrm{H}\right), 3.71$ (ddd, $J_{1} 11.60 \mathrm{~Hz}$, $\left.J_{2} 5.30 \mathrm{~Hz}, J_{3} 1.60 \mathrm{~Hz}, 1 \mathrm{H}\right) ;{ }^{13} \mathrm{C} \mathrm{NMR}\left(100 \mathrm{MHz}, \mathrm{CDCl}_{3}\right)$ $\delta 14.1,18.7,21.7,22.5,30.4,37.8,38.4,43.5,64.6,74.3$, 80.1, 170.3; (CAS 723340-91-8).

\section{2-Ethyl-4,7-dimethyloctahydro-2H-chromen-4-ol (15)}

Diastereoisomeric mixture: Eluent: hexane/ethyl acetate (4:1); colorless oil, yield $49 \%$; ${ }^{1} \mathrm{H}$ NMR $\left(400 \mathrm{MHz}, \mathrm{CDCl}_{3}\right) \delta$ 0.85-1.25 (m, 15H), 1.35-1.50 (m, $3 \mathrm{H}), 1.51-1.63(\mathrm{~m}, 2 \mathrm{H}), 1.68-1.82(\mathrm{~m}, 5 \mathrm{H}), 1.87-1.99(\mathrm{~m}$, $3 \mathrm{H}), 3.07\left(\mathrm{td}, J_{1} 10.44 \mathrm{~Hz}, J_{2} 4.24 \mathrm{~Hz}, 1 \mathrm{H}\right), 3.24-3.30(\mathrm{~m}$, $1 \mathrm{H}) ;{ }^{13} \mathrm{C}$ NMR $\left(100 \mathrm{MHz}, \mathrm{CDCl}_{3}\right) \delta 9.9,21.4,22.2,23.1$, 29.1, 31.5, 34.5, 41.6, 48.0, 52.4, 70.8, 75.9, 76.9. (CAS 134869-45-7).

\section{2-Isopropyl-4,7-dimethyloctahydro-2H-chromen-4-ol (16)}

Diastereoisomeric mixture: Eluent: hexane/ethyl acetate (7:1); colorless oil, yield 66\%; ${ }^{1} \mathrm{H} \mathrm{NMR}\left(400 \mathrm{MHz}, \mathrm{CDCl}_{3}\right.$ ) $\delta$ 0.84-1.09 (m, 14H), 1.12-1.21 (m, 4H), 1.35-1.50 (m, $2 \mathrm{H}), 1.61-1.75(\mathrm{~m}, 5 \mathrm{H}), 1.85-1.99(\mathrm{~m}, 3 \mathrm{H}), 3.00-3.10(\mathrm{~m}$, $2 \mathrm{H}) ;{ }^{13} \mathrm{C}$ NMR (100 MHz, $\left.\mathrm{CDCl}_{3}\right) \delta 18.3(2 \mathrm{C}), 21.0,25.8$, 26.2, 31.5, 32.5, 34.6, 36.5, 40.7, 42.3, 76.0, 78.3, 80.9. (CAS 134869-70-8)

\section{Acknowledgments}

Fapesp (05/59572-7), CNPq, CAPES for financial support and Luciana Vizotto (LNMR/Universidade Federal de São Carlos) to perform the NMR and nOe experiments.

\section{References}

1. Olier, C.; Kaafarani, M.; Gastaldi, S.; Bertrand, M. P.; Tetrahedron 2010, 66, 413; Vascondellos, M. L. A. A.; Miranda, L. S. M. A.; Quim. Nova 2006, 29, 834; Crosby, S. R.; Harding, J. R.; King, C. D.; Parker, G. D.; Willis, C. L.; Org. Lett. 2002, 4, 577; Hiebel, M. A.; Pelotier, B.; Piva, O.; Tetrahedron 2007, 63, 7874; Yadav, J. S.; Reddy, B. V. S.; Maity, T.; Kumar, G. G. K. S.; Tetrahedron Lett. 2007, 48, 8874; Chan, K. P.; Seow, A. H.; Loh, T. P.; Tetrahedron Lett. 2007, 48, 37; Yadav, J. S.; Reddy, B. V. S.; Maity, T.; Kumar, G. G. K. S. N.; Tetrahedron Lett. 2007, 48, 7155; Yadav, J. S.; Reddy, B. V. S.; Kumar, G. G. K. S. N.; Swamy, T.; Tetrahedron Lett. 2007, 48, 2205; Yadav, J. S.; Reddy, B. V. S.; Kumar, G. M.; Murthy, C. V. S. R.; Tetrahedron Lett. 2001, 42, 89.

2. Reddy, U. C.; Bondalapati, S.; Saikia, A. K.; J. Org. Chem. 2009, 74, 2605; Perron, F.; Albizati, K. F.; J. Org. Chem. 1987, 52, 4130; Clarke, P. A.; Santos, S.; Eur. J. Org. Chem. 2006, 2045, and references cited therein; Class, Y. J.; DeShong, P.; Chem. Rev. 1995, 95, 1843; Kopecky, D. J.; Rychnovsky, S. D.; J. Am. Chem. Soc. 2001, 123, 8420; Wang, Y.; Janjic, J.; Kozmin, S. A.; J. Am. Chem. Soc. 2002, 124, 13670; Tian, X. T.; Jaber, J. J.; Rychnovsky, S. D.; J. Org. Chem. 2006, 71, 3176; Hu, Y.; Skalitzky, D. J.; Rychnovsky, S. D.; Tetrahedron Lett. 1996, 37, 8679; Arundale, E.; Mikeska, L. A.; Chem. Rev. 1952, 51, 505; Miles, R. B.; Davis, C. E.; Coates, R. M.; J. Org. Chem. 2006, 71, 1493; Yadav, J. S.; Reddy, B. V. S.; Kumar, G. G. K. S. N.; Reddy, G. M.; Tetrahedron Lett. 2007, 48, 4903.

3. Gupta, P.; Sethi, V. K.; Taneja, S. C.; Shah, B. A.; Andotra, S. S.; Koul, S.; Chimni, S. S.; Oazi, G. N.; Helv. Chim. Acta 2007, 90, 196.

4. Abate, A.; Brenna, E.; Fronza, G.; Fuganti, C.; Gatti, F. G.; Serra, S.; Zardoni, E.; Helv. Chim. Acta 2004, 87, 765.

5. Alfonsi, K.; Colberg, J.; Dunn, P. J.; Fevig, T.; Jennings, S.; Johnson, T. A.; Kleine, H. P.; Knight, C.; Nagy, M. A.; Perry, D. A.; Stefaniak, M.; Green Chem. 2008, 10, 31; Abaee, M. S.; Mojtahedi, M. M.; Forghani, S.; Ghandchi, N. M.; Forouzani, M.; Sharifi, R.; Chaharnazm, B.; J. Braz. Chem. Soc. 2009, 20, 1895; Lenardão, E. J.; Trecha, D. O.; Ferreira, P. C.; Jacob, R. G.; Perin, G.; J. Braz. Chem. Soc. 2009, 20, 93; Bueno, M. A.; Silva, L. R. S. P.; Corrêa, A. G.; J. Braz. Chem. Soc. 2008, 19, 1264; Almeida, Q. A. R.; Pereira, M. L. O.; Coelho, R. B.; Carvalho, E. M.; Kaiser, C. R.; Jones Jr., J.; Silva, F. M.; J. Braz. Chem. Soc. 2008, 19, 894. 
6. Reddy, B. M.; Sreekanth, P. M.; Lakshmanan, P.; J. Molec. Catal. A: Chem. 2006, 237, 93; Bartoli, G.; Bartolacci, M.; Bosco, M.; Foglia, G.; Giuliani, A.; Marcantoni, E.; Sambri, L.; Torregiani, E.; J. Org. Chem. 2003, 68, 4594; Bartoli, G.; Bartolacci, M.; Giuliani, A.; Marcantoni, E.; Massaccesi, M.; Torregiani, E.; J. Org. Chem. 2005, 70, 169; Bartoli, G.; Bosco, M.; Giuli, S.; Giuliani, A.; Lucarelli, L.; Marcantoni, E.; Sambri, L.; Torregiani, E.; J. Org. Chem. 2005, 70, 1941; Bartoli, G.; Fernández-Bolaños, J. G.; Di Antonio, G.; Foglia, G.; Giuli, S.; Gunnella, R.; Mancinelli, M.; Marcantoni, E.; Paoletti, M.; J. Org. Chem. 2007, 72, 6029; Chen, J.-X.; Liu, M.-C.; Yang, X.-L.; Ding, J.-C.; Wu, H.-Y.; J. Braz. Chem. Soc. 2008, 19, 877; Costa, J. S.; Pisoni, D. S.; Silva, C. B.; Petzhold, C. L.; Russowsky, D.; Ceschi, M. A.; J. Braz. Chem. Soc. 2009, 20, 1448.

7. Sprecker, M. A.; Belko, R. P.; Hanna, M. R.; Beck, C. E. J.; Brucato, S. M.; US pat. 4,999,439 1991. (pp. 48).
8. Dos Santos, A. A.; Brito Jr., G. A.; Archilha, M. V. L.; Bele, T. G. A.; Dos Santos, G. P.; De Mello, M. B. M.; J. Braz. Chem. Soc. 2009, 20, 42.

9. Zukerman-Schpector, J.; Dos Santos, A. A.; Macedo, A.; Wendler, E. P.; Brito Jr., G. A.; Tiekink, E. R. T.; Z. Kristallogr. 2008, 223, 471.

10. The crystals of $\mathbf{7 f}$ are orthorhombic, space group $P 2_{1} 2_{1} 2_{1}$, with $a=5.5714(10) \AA, b=11.0182(12) \AA, c=18.753(3) \AA$, $V=1151.2(3) \AA^{3}, D_{x}=1.144 \mathrm{~g} \mathrm{~cm}^{-3}$, and $Z=4$. The structure was solved by direct-methods and refined by full-matrix leastsquares to final $R=0.050$. Tiekink, E. R. T.; Macedo, A.; Wendler, E. P.; Dos Santos, A. A.; Zukerman-Schpector, J.; Acta Cryst. 2010, E66, o1233.

Received: January 22, 2010 Web Release Date: May 4, 2010

FAPESP has sponsored the publication of this article. 